\title{
Desempeño productivo y rendimiento de canal en pavos alimentados con harina de plumas tratadas con $\mathrm{NaOH}$
}

\section{Productive performance and carcass yield of turkey fed feather meal treated with $\mathrm{NaOH}$}

\author{
Enrique Loyra T, ${ }^{1}$ M.Sc, Ronald Santos R, ${ }^{1 *}$ Ph.D, Luis Sarmiento F, ${ }^{1}$ Ph.D, \\ José Segura $\mathrm{C}^{1}{ }^{1} \mathrm{Ph} . \mathrm{D}$.
} Universidad de Yucatán, Campus de Ciencias Biológicas y Agropecuarias, Departamento de Nutrición
Animal, Apartado Postal 4-116, Itzimná, Yucatán, México. *Correspondencia: rsantos@uady.mx.

Recibido: Agosto de 2011; Aceptado: Enero de 2013.

\section{RESUMEN}

Objetivo. Evaluar el desempeño productivo y el rendimiento de la canal en pavos en crecimiento alimentados con dietas elaboradas con harina de plumas (HP). Materiales y métodos. Los tratamientos fueron una dieta control y dos dietas experimentales con harina de plumas tratada con 50 ó $100 \mathrm{~g}$ de $\mathrm{NaOH} / \mathrm{kg}$. Se utilizó un diseño de bloques al azar. El consumo de alimento y el peso de los animales se registró cada dos semanas. Los datos del desempeño productivo se analizaron con el procedimiento MIXED del programa estadístico SAS. El rendimiento de la canal se analizó con el procedimiento GLM del programa estadístico SAS. Resultados. Los pavos que consumieron la dieta testigo tuvieron mejores ganancias de peso (GP), consumo de alimento (CA), peso de la canal y de sus partes $(p<0.05)$ que aquellas con harina de plumas. Sin embargo, se observó una mayor GP, CA, peso de la canal y del muslo $(p<0.05)$ cuando se trató la harina de plumas con $100 \mathrm{~g}$ de $\mathrm{NaOH} / \mathrm{kg}$. Conclusiones. Los resultados obtenidos indican que la utilización de harina de plumas disminuyó el comportamiento productivo y el rendimiento de canal en los pavos. Sin embargo, el aumento del tratamiento de la harina de plumas de 50 a $100 \mathrm{~g}$ de NaOH/ kg mejoró el comportamiento productivo y el rendimiento de canal.

Palabras clave: Alimentación animal, composición de la canal, harina de plumas, hidróxido de sodio, pavos (Fuente:AIMS). 


\section{ABSTRACT}

Objective. Evaluate the productive performance and carcass yield of growing turkeys fed with diets including feather meal. Materials and methods. The treatments were: a control diet and two diets with feather meal (FM) treated with 50 or $100 \mathrm{~g}$ of $\mathrm{NaOH} / \mathrm{kg}$ ( $5 \%$ inclusion) each. A random block design was used. Feed consumption and weight gain were recorded every two weeks. Productive performance data was analyzed using the MIXED procedure of the SAS statistical program. Carcass yield was analyzed using the GLM procedure of the SAS statistical program. Results. Weight gain (WG), feed consumption (FC), carcass yield and composition were higher in turkeys fed with the control diet, in comparison to those fed with FM diets $(p<0.05)$. However, Turkeys fed diets with 100 $\mathrm{g} \mathrm{NaOH} / \mathrm{kg}$ presented higher WG, FC, carcass yield and thigh weight $(p<0.05)$ than turkeys fed diets with $50 \mathrm{~g} \mathrm{NaOH} / \mathrm{kg}$. Conclusions. The use of FM reduces the productive performance and carcass yield in turkeys. However, improvement on productivity and carcass yield were observed when FM treatment with $\mathrm{NaOH}$ increased from 50 to $100 \mathrm{~g} / \mathrm{kg}$.

Key words: Animal feeding, feather meal, sodium hydroxide, carcass composition, turkeys (Source: AIMS).

\section{INTRODUCCIÓN}

La harina de plumas (HP) ha sido objeto de interés en nutrición animal debido a su elevado contenido de proteína cruda (PC) $82.1 \%$ (1). Sin embargo, la digestibilidad de la proteína cruda es muy baja ( $4.67 \%)$ debido a la gran cantidad de puentes di-sulfuro de cistina que tiene en su estructura la queratina, principal proteína del ingrediente (2). En países en desarrollo, la situación es más complicada, pues las plumas al no tener ninguna utilidad en la alimentación animal, se desechan de forma inadecuada, y se vuelven una fuente de contaminación del medio ambiente.

Diversos métodos de procesamiento se han aplicado para incrementar la digestibilidad de la $\mathrm{HP}$, siendo el más común la cocción con vapor a alta presión en autoclave, con variaciones en la temperatura, humedad, presión y tiempo de duración del proceso. Con este tratamiento se han obtenido resultados aceptables en la digestibilidad in vitro de la proteína cruda $(59.2 \%)(3)$.

Otros tratamientos mas recientes que han demostrado ser efectivos, son los procesos que involucran el uso de enzimas que degradan la queratina de las plumas. Con esta biotecnología se ha logrado degradar mas del $90 \%$ de las plumas después de incubarla con cepas de bacterias productoras de queratinasas (4). Sin embargo, estos tratamientos representan un alto costo energético y económico.

Por lo tanto, resulta de interés evaluar otros procesos más económicos y sencillos que permitan aprovechar el potencial nutricional de la HP. Por ejemplo, el tratamiento entre 2 y 24 horas de la HP con una solución de $\mathrm{NaOH}$ al $56.2 \%$, puede solubilizar entre 30 y $79 \%$ del nitrógeno, respectivamente (2). Sin embargo, el alto contenido de sodio en las plumas como resultado del tratamiento, pudiera restringir su incorporación en el alimento de aves.

Existe poca información disponible sobre el desempeño productivo de animales monogástricos alimentados con HP. En la mayoría de estos trabajos las plumas fueron sometidas a un proceso de cocción con vapor de agua a alta presión en autoclave. En cerdos, se observó una reducción en la ganancia de peso y eficiencia alimenticia cuando se incluye la HP hasta un $10 \%$ en la dieta. Sin embargo, hasta un $8 \%$ no tuvo efecto sobre la ganancia diaria de tejido magro (5). Una dieta hasta con $5 \%$ de inclusión de HP utilizada en gallinas ponedoras no afectó el desempeño productivo y la calidad del huevo (6). En pollos se ha reportado que la digestibilidad ileal de los aminoácidos de la HP es muy baja, en comparación con la pasta de soya (7). El comportamiento productivo de las aves podría verse afectado si no se toma en consideración este punto. Al respecto se ha observado que el desempeño productivo de los cerdos no se afecta cuando la dieta con harina de plumas se suplementa con la cantidad requerida de aminoácidos (8).

Con base en lo anterior se planteó el presente trabajo, cuyo objetivo fue evaluar el efecto de utilizar $\mathrm{HP}$, tratadas con $\mathrm{NaOH}$ a dos concentraciones diferentes en dietas de pavos en crecimiento, desempeño productivo y rendimiento de la canal. 


\section{MATERIALES Y MÉTODOS}

Sitio de estudio. El experimento se llevó a cabo en las instalaciones del área de Nutrición Animal del Campus de Ciencias Biológicas y Agropecuarias (CCBA) de la Universidad Autónoma de Yucatán, ubicada en el km 15.5 de la carretera Mérida-Xmatkuil $\left(20^{\circ} 58^{\prime} \mathrm{N}\right.$ y $\left.89^{\circ} 37^{\prime} \mathrm{O}\right)$. El clima de la región es cálido y húmedo, con un rango de temperatura media anual de 24.0 a $28.0^{\circ} \mathrm{C}$ y un rango de precipitación total anual de 800 a 1000 mm (9).

Tratamientos. Las dietas utilizadas consistieron en un alimento testigo a base de sorgo molido y pasta de soya y un alimento experimental con $5 \%$ de inclusión de harina de plumas (HP) tratadas con 50 ó $100 \mathrm{~g} / \mathrm{kg}$ de $\mathrm{NaOH}$, respectivamente (Tabla 1 ). La inclusión del 5\% de HP representó una sustitución de alrededor del $23 \%$ de la proteína cruda de la dieta. Todas las dietas se proporcionaron ad libitum, en forma de harina, durante las 6 semanas que duró el periodo experimental. Para el balanceo de las raciones, se utilizaron los lineamientos de las tablas de ingredientes y requerimientos nutricionales del National Reseach Council (NRC) (10), así como el contenido de aminoácidos de la HP mencionada por Barbour et al (11). Cabe mencionar que la cantidad de sal en las dietas se ajustó de acuerdo con el nivel de sodio que aportaba el $\mathrm{NaOH}$ aplicado a las HP (Tabla 1). Se utilizó 0.1 ó $0 \%$ de sal $(\mathrm{NaCl})$ en la dieta con HP tratada con 50 ó $100 \mathrm{~g} \mathrm{NaOH} / \mathrm{kg}$ de HP respectivamente, para mantener el nivel de sodio en la dieta dentro de los valores recomendados por el NRC (10).

Preparación de HP. Las plumas se obtuvieron de un matadero comercial de pavos, las cuales fueron secadas al sol durante dos días. Después de este tratamiento las plumas presentaron un $90.4 \%$ de materia seca. Posteriormente, las plumas fueron tratadas en lotes de $10 \mathrm{~kg}$ y rociadas con una solución con 0.5 ó $1 \mathrm{~kg}$ de $\mathrm{NaOH}$ en $20 \mathrm{~L}^{-1}$ de agua, para obtener la pluma tratada a utilizar. Después de $24 \mathrm{~h}$ se procedió al secado de las HP en una estufa de aire forzado a $60^{\circ} \mathrm{C}$ por $48 \mathrm{~h}$. Finalmente, se molieron las plumas en un molino de martillos con criba de $3 \mathrm{~mm}$.

Animales. Se utilizaron 82 pavos machos Nicholas-700 de 10 semanas de edad distribuidos en dos bloques. El trabajo experimental con el primer bloque se realizó de septiembre a octubre y con el segundo de noviembre a diciembre. El peso inicial de los pavos fue de $6.04 \pm 0.36 \mathrm{~kg}^{-1}$ y $6.43 \pm 0.44 \mathrm{~kg}^{-1}$ en el primer y segundo bloque respectivamente. En las dietas con $\mathrm{HP}$, se utilizaron 2 aves por unidad experimental y 9 repeticiones por tratamiento en cada bloque, mientras que para la dieta testigo hubo 2 repeticiones en el primer bloque y 3 en el segundo. En total hubo 18 repeticiones por cada tratamiento con HP y 5 para el control. Se utilizaron mayor numero de repeticiones para los tratamientos con HP, pues es donde se esperaba un mayor coeficiente de variación. Las aves fueron alojadas en una caseta avícola provista con jaulas de $1 \mathrm{~m}^{2}$ de superficie cada una, con piso de concreto cubierto con viruta de madera, un comedero colgante tipo tolva y bebedero automático.

Comportamiento productivo. En cada bloque, los pavos fueron pesados al inicio del experimento y a las 2,4 y 6 semanas posteriores para calcular la ganancia de peso. Asimismo, en el mismo periodo se registró el consumo de alimento. La eficiencia alimenticia se calculó dividiendo la ganancia de peso entre el consumo de alimento.

Rendimiento de canal. Al final de cada periodo experimental en cada bloque, un ave de cada corral fue seleccionada al azar y sacrificada para medir el rendimiento de canal. Previo al sacrificio los pavos se sometieron a un ayuno de 12 horas. El sacrificio consistió en aturdimiento con descarga eléctrica y posterior corte de la vena yugular para provocar desangrado. Las aves fueron escaldadas a una temperatura de $60^{\circ} \mathrm{C}$ por 1 minuto, posteriormente fueron desplumadas y evisceradas manualmente eliminando de la canal la cabeza, cuello, vísceras abdominales y patas. Se registró el peso de la canal caliente y de la mitad derecha se obtuvieron los cortes principales (pechuga, ala, muslo y pierna).

Análisis estadístico. Se utilizó un diseño experimental de bloques al azar debido a que solo se disponía de 20 jaulas en el primer bloque y de 21 en el segundo bloque con la intención de incrementar el numero de repeticiones en los tratamientos. Los datos de ganancia de peso, consumo de alimento y eficiencia alimenticia se analizaron con el procedimiento MIXED para medidas repetidas del programa estadístico SAS (12). En tanto que los datos de peso inicial y peso final, rendimiento de canal y peso de los cortes principales se analizaron con el procedimiento GLM del programa SAS (12). Se realizaron comparaciones entre las medias de los tratamientos, por medio del procedimiento de mínima diferencia significativa, cuando fue necesario. 


\section{RESULTADOS}

Se puede observar en la tabla 1 la composición y el análisis quimico de las dietas utilizadas.

Tabla 1. Composición de ingredientes y análisis químico calculada de las dietas utilizadas.

\begin{tabular}{lcc}
\hline & \multicolumn{2}{c}{ Dietas } \\
\cline { 2 - 3 } Ingredientes (\%) & Testigo & Experimental \\
\hline Sorgo molido & 58.2 & 66.8 \\
Pasta de Soya & 33.4 & 21.6 \\
Aceite de Soya & 3.7 & 2.2 \\
Harina de plumas ${ }^{1}$ & -- & 5.0 \\
Ortofosfato ${ }^{2}$ & 2.3 & 2.4 \\
CaCO3 38\% $_{\text {Sal }}$ & 1.5 & 1.4 \\
Flavomicyn 4\% & 0.4 & 0.1 ó 0.0 \\
Minerales & 0.05 \\
Metionina 82\% & 0.05 & 0.05 \\
Lisina HCl & 0.05 & 0.11 \\
Vitaminas & & 0.28 \\
Análisis calculado (\%) & 0.06 & 0.03 \\
\hline PC & 0 & 20 \\
Ca & 0.03 & 1.15 \\
P total & 20.0 & 0.8 \\
Lisina & 1.15 & 1.0 \\
Metionina & 0.8 & 0.35 \\
Met + Cis & 1.03 & 0.87 \\
EM (Mcal/kg) & 0.35 & 2.99 \\
\hline
\end{tabular}

${ }^{1} 50$ ó $100 \mathrm{~g} \mathrm{NaOH} / \mathrm{kg} \mathrm{BF}$ de HP.

$220 \%$ de $\mathrm{P}$ y $20 \%$ de Ca.

${ }^{3}$ Se utilizó 0.1 ó $0.0 \mathrm{~kg}$ de sal en la dieta con HP tratada con 50 y $100 \mathrm{~g}$ $\mathrm{NaOH} / \mathrm{kg}$ de $\mathrm{HP}$, respectivamente.

${ }^{4}$ Por kg de dieta: manganeso, $65 \mathrm{mg}$; yodo, $1 \mathrm{mg}$; hierro, $55 \mathrm{mg}$; cobre, $6 \mathrm{mg}$; zinc, $55 \mathrm{mg}$; selenio, $0.3 \mathrm{mg}$.

${ }^{5}$ Por kg de dieta: vit. A, 2000 UI; vit. D, 625 UI; vit. E, 2 UI; vit.K, 0.5 $\mathrm{mg}$; vit. B12, $0.0005 \mathrm{mg}$; riboflavina, $1.375 \mathrm{mg}$; pantotenato de calcio, $3.25 \mathrm{mg}$; niacina, $9 \mathrm{mg}$; colina, $125 \mathrm{mg}$; ácido fólico, $0.125 \mathrm{mg}$; tiamina, $0.25 \mathrm{mg}$; piridoxina, $0.55 \mathrm{mg}$; biotina, 0.0125 .

En la tabla 2, se presenta el análisis proximal de la HP cruda y de la hidrolizada con $\mathrm{NaOH}$. Se puede observar una reducción en el contenido de PC de 89.7 y 90.5 en la harina de plumas sin tratar y tratada con $50 \mathrm{~g}$ de $\mathrm{NaOH}$ a $85.7 \%$ en la HP tratada con $100 \mathrm{~g} \mathrm{NaOH}$. También, se observo una reducción de la FC $(1.5,0.9$ y $0.6 \%$, para la harina de plumas sin tratar, $50 \mathrm{~g} \mathrm{NaOH}$ y $100 \mathrm{~g} \mathrm{NaOH}$, respectivamente) y un aumento del contenido de cenizas conforme aumento la cantidad de $\mathrm{NaOH}$ utilizada $(3.5,8.9$ y $14.7 \%$ para la harina de plumas sin tratar, $50 \mathrm{~g} \mathrm{NaOH}$ y $100 \mathrm{~g} \mathrm{NaOH}$, respectivamente).

Tabla 2. Composición química de la harina de plumas tratada con $\mathrm{NaOH}$ ó sin tratar.

\begin{tabular}{|c|c|c|c|}
\hline \multirow{2}{*}{$\begin{array}{c}\text { Composición } \\
(\%)\end{array}$} & \multicolumn{3}{|c|}{ Harina de plumas } \\
\hline & Sin tratar & $50 \mathrm{~g} \mathrm{NaOH} / \mathrm{kg}$ & $100 \mathrm{~g} \mathrm{NaOH} / \mathrm{kg}$ \\
\hline Materia Seca & 92.3 & 93.0 & 94.1 \\
\hline Proteína Cruda & 89.7 & 90.5 & 85.7 \\
\hline Fibra Cruda & 1.5 & 0.9 & 0.6 \\
\hline Extracto Etéreo & 0.6 & 0.8 & 0.8 \\
\hline Cenizas & 3.5 & 8.9 & 14.7 \\
\hline
\end{tabular}

En la tabla 3 se presentan los resultados del desempeño productivo de los pavos. Se encontró que el peso final, la ganancia de peso y el consumo de alimento $(p<0.05)$ de los pavos alimentados con la dieta testigo fueron significativamente superiores a la de aquellos que consumieron las dietas con HP. Sin embargo, el peso final, la ganancia de peso y el consumo de alimento fueron mayores en los pavos que consumieron la HP tratada con $100 \mathrm{~g}$ de $\mathrm{NaOH} / \mathrm{kg}(p>0.05)$ en comparación con los que consumieron la dieta con la HP tratada con $50 \mathrm{~g}$ de $\mathrm{NaOH} / \mathrm{kg}(13.0,10.9$ y $12.1 \mathrm{~kg}$ de peso; $165.8,127.0$ y $144 \mathrm{~g} /$ día de ganancia de peso y $722.8,574.5$ y $648.8 \mathrm{~g} /$ día de consumo, para la dieta control, $50 \mathrm{~g} \mathrm{NaOH}$ y $100 \mathrm{~g} \mathrm{NaOH}$, respectivamente). En la variable eficiencia alimenticia no se encontró diferencia significativa entre los tratamientos, $(p>0.05)$.

Tabla 3. Desempeño productivo de pavos alimentados con HP tratada con $\mathrm{NaOH}$.

\begin{tabular}{|c|c|c|c|c|}
\hline \multirow[b]{2}{*}{ Variables } & \multicolumn{3}{|c|}{ Tratamientos } & \multirow[b]{2}{*}{ EEM } \\
\hline & Testigo & $50 \mathrm{~g} \mathrm{NaOH}$ & $100 \mathrm{~g} \mathrm{NaOH}$ & \\
\hline$n$ & 5 & 18 & 18 & \\
\hline Peso inicial (kg) & 6.2 & 6.2 & 6.2 & 0.07 \\
\hline Peso final (kg) & $13.0^{\mathrm{a}}$ & $10.9^{c}$ & $12.1^{\mathrm{b}}$ & 1.33 \\
\hline $\begin{array}{l}\text { Ganancia de peso } \\
\text { (g/dia) }\end{array}$ & $165.8^{\mathrm{a}}$ & $127.0^{c}$ & $144.0^{\mathrm{b}}$ & 7.20 \\
\hline $\begin{array}{l}\text { Consumo de } \\
\text { alimento (g/dia) }\end{array}$ & $722.8^{\mathrm{a}}$ & $574.5^{c}$ & $648.8^{\mathrm{b}}$ & 32.99 \\
\hline $\begin{array}{l}\text { Eficiencia } \\
\text { alimenticia (g/g) }\end{array}$ & 0.23 & 0.22 & 0.22 & 0.008 \\
\hline
\end{tabular}

Medias con diferente literal en la misma línea son diferentes estadísticamente $(p<0.05)$.

En la tabla 4 se presentan los resultados de rendimiento de canal. Se observa que los pavos que consumieron la dieta testigo tuvieron mayor peso de canal $(10.2 \mathrm{~kg})$, de pechuga $(2.9 \mathrm{~kg})$, de ala $(1.3 \mathrm{~kg})$, de muslo $(1.6 \mathrm{~kg})$ y de pierna $(1.5 \mathrm{~kg})$ en comparación a los de las dietas con HP $(p<0.05)$. No obstante, el peso de la canal y del muslo fueron significativamente superiores $(p<0.05)$ en los pavos de la dieta con HP tratada con $100 \mathrm{~g} \mathrm{NaOH} / \mathrm{kg}(9.0 \mathrm{y}$ $1.5 \mathrm{~kg}$, respectivamente), en comparación a los de la dieta con $50 \mathrm{~g} \mathrm{NaOH} / \mathrm{kg}(8.4$ y 1.4 $\mathrm{kg}$, respectivamente). Sin embargo, cuando se expreso el peso de la canal y de los cortes principales como porcentaje del peso vivo al sacrificio no se encontraron diferencias significativas entre tratamientos $(p>0.05)$. 
Tabla 4. Peso y rendimiento de canal y de los cortes principales de pavos alimentados con HP tratada con $\mathrm{NaOH}$

\begin{tabular}{|c|c|c|c|c|}
\hline \multirow{2}{*}{ Peso (kg) } & \multicolumn{3}{|c|}{ Tratamientos } & \multirow{2}{*}{ EEM } \\
\hline & Testigo & $50 \mathrm{~g} \mathrm{NaOH}$ & $100 \mathrm{~g} \mathrm{NaOH}$ & \\
\hline $\mathrm{n}$ & 5 & 9 & 9 & \\
\hline Canal & $10.2^{\mathrm{a}}$ & $8.4^{c}$ & $9.0^{\mathrm{b}}$ & 0.33 \\
\hline Pechuga & $2.9^{\mathrm{a}}$ & $2.2^{\mathrm{b}}$ & $2.4^{\mathrm{b}}$ & 0.15 \\
\hline Ala & $1.3^{\mathrm{a}}$ & $1.1^{\mathrm{b}}$ & $1.1^{\mathrm{b}}$ & 0.04 \\
\hline Muslo & $1.6^{\mathrm{a}}$ & $1.4^{\mathrm{c}}$ & $1.5^{\mathrm{b}}$ & 0.05 \\
\hline Pierna & $1.5^{\mathrm{a}}$ & $1.3^{\mathrm{b}}$ & $1.3^{\mathrm{b}}$ & 0.06 \\
\hline \multicolumn{5}{|c|}{ Rendimiento en proporción al peso vivo (\%) } \\
\hline Canal & 76.5 & 75.4 & 75.7 & 0.53 \\
\hline Pechuga & 28.4 & 26.2 & 26.3 & 0.96 \\
\hline Ala & 12.4 & 12.6 & 12.3 & 0.36 \\
\hline Muslo & 15.9 & 16.3 & 16.4 & 0.35 \\
\hline Pierna & 14.3 & 15.2 & 14.8 & 0.46 \\
\hline
\end{tabular}

Medias con diferente literal en la misma línea son diferentes estadísticamente $(p<0.05)$

\section{DISCUSIÓN}

La disminución de la ganancia de peso y del peso final $(p<0.05)$ de los pavos alimentados con HP estuvo directamente asociada al menor consumo de alimento observado en estos tratamientos $(p<0.05)$, ya que la eficiencia alimenticia no difirió entre los tratamientos $(p>0.05)$. Los resultados indican que las dietas con HP se aprovecharon igual que la dieta testigo. Lo observado en este trabajo contrasta con lo reportado por Barbour et al (11) quienes observaron que la conversión alimenticia mejoro en pollos de engorda cuando sometieron las HP a un proceso de digestión enzimática y de cocción con vapor de agua a alta presión en autoclave. Estas diferencias pueden deberse al hecho de que en el presente trabajo las HP se sometieron a hidrólisis química con $\mathrm{NaOH}$, en vez de hidrolisis enzimática. Además, en el trabajo de Barbour et al (11) las HP se cocieron con vapor de agua a alta presión en autoclave, lo que favorece la coagulación de las proteínas y mejora su digestión.

La reducción del consumo de alimento en las dietas con $\mathrm{HP}$, en comparación con el tratamiento testigo probablemente se deba a que el tratamiento con $\mathrm{NaOH}$, no fue muy efectivo para reducir la voluminosidad de la HP, lo cual causo un efecto de llenado físico del tracto digestivo y la consecuente reducción del consumo de alimento. Lo anterior fue más evidente en las dietas donde la HP se trató con $50 \mathrm{~g} \mathrm{NaOH} / \mathrm{kg}$. Se ha reportado que las plumas de aves tienen poca densidad, lo que les confiere propiedades de voluminosidad. En este sentido, Moritz y Latshaw (3) reportaron que la densidad de la HP disminuyo y el contenido de fibra detergente ácida aumento conforme se redujo el grado de procesamiento físico por presión durante el procesamiento en autoclave. Estos autores, reportan que la densidad de la HP aumento de 375 a $584 \mathrm{~kg} / \mathrm{m}^{3}$, cuando la presión por vapor se incremento de 207 a $517 \mathrm{kPa}$. Simultáneamente, la fibra detergente acida de HP disminuyo de 57 a 13.9\%. En relación a lo anterior, se pudo observar que el tratamiento con $100 \mathrm{~g}$ de $\mathrm{NaOH}$ redujo en un $60 \%$ el contenido de fibra cruda de las plumas, con respecto a las plumas sin tratar y un 33\% con respecto a las plumas tratadas con $50 \mathrm{~g}$ de $\mathrm{NaOH}$ (Tabla 2). La reducción en fibra cruda de las plumas pudo contribuir a que la dieta con HP tratada con $100 \mathrm{~g}$ de $\mathrm{NaOH}$ tuviera un mayor consumo en comparación a la dieta con $\mathrm{HP}$ tratada con $50 \mathrm{~g}$ de $\mathrm{NaOH}$.

Otros autores mencionan que la disminución en el consumo de alimento y ganancia de peso cuando se incluye HP en las dietas puede atribuirse a la pobre digestibilidad de su PC y al desbalance en el contenido de sus aminoácidos. La eficiencia alimenticia también se puede reducir por efecto de estos factores $(8,13)$. Sin embargo, en este trabajo no se encontraron diferencias en la eficiencia alimenticia entre los tratamientos $(p>0.05)$, lo que sugiere que el aprovechamiento de las dietas fue similar.

Los datos del desempeño productivo indican que el tratamiento de las $\mathrm{HP}$ con $\mathrm{NaOH}$ no fue suficiente para mejorar el consumo de las dietas hasta un nivel similar al del tratamiento testigo. Sin embargo, el mayor consumo de alimento y ganancia de peso de los pavos en la dieta con HP tratada con $100 \mathrm{~g}$ de $\mathrm{NaOH}$, en relación con la dieta con $50 \mathrm{~g}$ de $\mathrm{NaOH}(\mathrm{p}<0.05)$ sugiere una mejora en sus características físicas, lo cual podría estar relacionado con una mayor ruptura de enlaces di-sulfuro de la queratina. En este sentido, Kim et al (2) encontraron que al tratar plumas con $\mathrm{NaOH}$, la digestibilidad en pepsina de la HP como sustrato se incrementó. Se requieren mas estudios para evaluar y confirmar el efecto del $\mathrm{NaOH}$ sobre las características físicas y químicas de la HP y su efecto sobre el consumo de alimento en pavos.

El menor peso de la canal y cortes principales $(p<0.05)$ en los pavos de los tratamientos con HP comparado con el testigo, esta asociado al menor peso al finalizado que alcanzaron los pavos $(p<0.05)$ como consecuencia del menor consumo de alimento registrado durante la prueba $(p<0.05)$. Asimismo, el incremento del peso de la canal y del muslo $(p<0.05)$ en 
el tratamiento de la $\mathrm{HP}$ con $100 \mathrm{~g}$ de $\mathrm{NaOH} /$ $\mathrm{kg}$, estuvo relacionado a un mayor consumo de alimento y ganancia de peso $(p<0.05)$ observado en los pavos de este tratamiento, en contraste con lo ocurrido en los pavos del tratamiento con 50 de $\mathrm{NaOH} / \mathrm{kg}$. Diversos estudios confirman el efecto negativo que tiene la disminución en consumo de PC y de aminoácidos esenciales sobre el crecimiento de las aves y sobre el peso de la canal y de los cortes principales (14-17). Sin embargo, cuando se evaluó el rendimiento de la canal y de los cortes principales como proporción del peso vivo al sacrificio, no se encontraron diferencias significativas entre tratamientos $(p<0.05)$, lo que indica que no hubo crecimiento diferencial entre los cortes principales por efecto de los tratamientos.
En conclusión la utilización de HP hidrolizada con $\mathrm{NaOH}$ en las dietas de pavos afectó negativamente el desempeño productivo y el rendimiento de canal de los pavos. Sin embargo, el tratamiento de la HP con $100 \mathrm{~g}$ de $\mathrm{NaOH}$ mejoró el comportamiento productivo y el rendimiento de canal, en comparación con el tratamiento con $50 \mathrm{~g}$ de $\mathrm{NaOH}$. Estos resultados permiten pensar en la posibilidad de evaluar en trabajos posteriores el uso de cantidades superiores a los $100 \mathrm{~g}$ de $\mathrm{NaOH}$ para hidrolizar las HP y el efecto complementario de la cocción con vapor de agua a alta presión en autoclave.

\section{REFERENCIAS}

1. Xilong L, Reza R, Peng L, Guoyao W. Composition of amino acids in feed ingredients for animal diets. Amino Acids 2011; 40:1159-1168.

2. Kim WK, Lorenz ES, Patterson PH. Effect of enzymatic and chemical treatments on feather solubility and digestibility. Poult Sci 2002; 81:95-98.

3. Moritz JS, Latshaw JD. Indicators of nutritional value of hydrolyzed feather meal. Poult Sci $2001 ; 80: 79-86$.

4. Mazotto AM, Coelho RR, Cedrola SM, de Lima MF, Couri S, Paraguai de Souza E, Vermelho AB. Keratinase Production by Three Bacillus spp. Using Feather Meal and Whole Feather as Substrate in a Submerged Fermentation. Enzyme Res 2011; doi: $10.4061 / 2011 / 523780$.

5. Heugten van E, Kempen van TATG. Growth performance, carcass characteristics, nutrient digestibility and fecal odorous compounds in growing-finishing pigs fed diets containing hydrolyzed feather meal. J Anim Sci 2002; $80: 171-178$.

6. Senkoylu N, Samli HE, Akyurek H, Agma A, Yasar S. Performance and egg characteristics of laying hens fed diets incorporated with poultry by-product and feather meals. J Appl Poult Res 2005; 14:542-547.
7. Bandegan A, Kiarie E, Payne RL, Crow GH, Guenter W, Nyachoti CM. Standardized ileal amino acid digestibility in dry-extruded expelled soybean meal, extruded canola seed-pea, feather meal, and poultry byproduct meal for broiler chickens. Poult Sci 2010; 89:2626-2633.

8. Divakala KC, Chiba LI, Kamalakar RB, Rodning SP, Welles EG, Cummins KA, Swann J, Cespedes F, Payne RL. Amino acid supplementation of hydrolyzed feather meal diets for finisher pigs. J Anim Sci 2009; 87:270-1281

9. Instituto Nacional de Estadistica y Geografia (INEGI). Anuario estadistico de los Estados Unidos Mexicanos 2010. Aguascalientes, Ags: INEGI; 2010.

10. National Research Council. Nutrient requirements of domestic animals. Nutrient requirements of poultry. 9th Rev. Edition. Washington DC: National Academic Press; 1994

11. Barbour GW, Werling $M$, Yersin AG, Lilburn MS. The effect of enzime predigestion on the nutritional quality of prepressed turkey feather meal. Poul Sci 2002; 81:1032-1037.

12. SAS/STAT-Useŕ s Guide. Cary, NC, USA: SAS Inst. Inc; 2006. 
13. Peñaranda Ali F, Santos Ricalde R, Sarmiento Franco L, Segura Correa J, Gutierrez Triay M. Effect of dietary protein and lysine on performance and carcass yield of turkeys. Am J Anim Vet Sci 2010; 5:27-32.

14. Waibel PE, Carlson CW, Brannon JA, Noll SL. Limiting amino acids after methionine and lysine with growing turkeys fed low-protein diets. Poult Sci 2000; 79:1290-1298.

15. Waibel PE, Carlson CW, Brannon JA, Noll $\mathrm{SL}$. Identification of limiting amino acids in methionine and lysine supplemented lowprotein diets for turkeys. Poult Sci 2000; 79:1299-1305.
16. Tesseraud S, Pym RAE, Le Bihan-Duval E, Duclos MJ. Response of broilers selected on carcass quality to dietary protein supply: live performance, muscle development, and circulating insulin-like growth factors (IGF-I and -II). Poult Sci 2003; 82:1011-1016.

17. Lemme A, Frackenpohl UA, Petri A, Meyer $H$. Effects of reduced dietary protein concentrations with amino acid supplementation on performance and carcass quality in turkey toms 14 to 140 days of age. Int J Poult Sci 2004; 3:391-399. 